\title{
Psychiatric Disorders and Hydroxychloroquine for Coronavirus Disease 2019 (COVID-19): A VigiBase Study
}

\author{
Philippe Garcia ${ }^{1}$ - Alexis Revet ${ }^{2,3} \cdot$ Antoine Yrondi $^{4}$. Vanessa Rousseau ${ }^{1,3} \cdot$ Yannick Degboe $^{5}$. \\ François Montastruc ${ }^{1,3}$
}

Accepted: 30 September 2020 / Published online: 19 October 2020

(c) Springer Nature Switzerland AG 2020

\begin{abstract}
Introduction In the stressful context of the coronavirus disease 2019 (COVID-19) pandemic, some reports have raised concerns regarding psychiatric disorders with the use of hydroxychloroquine. In this study, we reviewed all psychiatric adverse effects with hydroxychloroquine in COVID-19 patients, as well as in other indications, reported in VigiBase, the World Health Organization's (WHO) global database of individual case safety reports.

Methods First, we analyzed all psychiatric adverse effects, including suicide, of hydroxychloroquine in COVID-19 patients reported to 16 June 2020 . We also performed disproportionality analysis to investigate the risk of reporting psychiatric disorders with hydroxychloroquine compared with remdesivir, tocilizumab, or lopinavir/ritonavir prescribed in COVID-19 patients. We used reporting odds ratios (RORs) and their 95\% confidence intervals (CIs) to calculate disproportionality. Second, we sought to examine the psychiatric safety profile of hydroxychloroquine in other indications (before 2020).

Results Among the 1754 reports with hydroxychloroquine in COVID-19 patients, we found 56 psychiatric adverse effects. Half of these adverse effects were serious, including four completed suicides, three cases of intentional self-injury, and 12 cases of psychotic disorders with hallucinations. Compared with remdesivir, tocilizumab, or lopinavir/ritonavir, the use of hydroxychloroquine was associated with an increased risk of reporting psychiatric disorders (ROR 6.27, 95\% CI 2.74-14.35). Before 2020, suicide was the main cause of death among all adverse drug reactions reported with hydroxychloroquine, followed by cardiac adverse effects (cardiomyopathy) and respiratory failure.

Conclusions This pharmacovigilance analysis suggests that COVID-19 patients exposed to hydroxychloroquine experienced serious psychiatric disorders, and, among these patients, some committed suicide. Further real-world studies are needed to quantify the psychiatric risk associated with hydroxychloroquine during the COVID-19 pandemic.
\end{abstract}

François Montastruc

francois.montastruc@univ-tlse3.fr

1 Department of Medical and Clinical Pharmacology, Centre of PharmacoVigilance and Pharmacoepidemiology, Faculty of Medicine, Toulouse University Hospital (CHU), 37 Allées Jules Guesde, 31000 Toulouse, France

2 Department of Child and Adolescent Psychiatry, Faculty of Medicine, Toulouse University Hospital (CHU), Toulouse, France

3 UMR 1027 Pharmacoepidemiology, Assessment of Drug Utilization and Drug Safety, INSERM, CIC 1426, University Paul Sabatier Toulouse, Toulouse, France

4 Department of Medical Psychiatry, Toulouse University Hospital (CHU), Faculty of Medicine, Treatment Resistant Depression Expert Center, FondaMental, ToNIC Toulouse NeuroImaging Center, University Paul Sabatier Toulouse, INSERM, Toulouse, France

5 Department of Rheumatology, Faculty of Medicine, Toulouse University Hospital (CHU), Toulouse, France

\section{Key Points}

The use of hydroxychloroquine for coronavirus disease 2019 (COVID-19) patients was associated with an increased risk of reporting psychiatric disorders.

These adverse effects ranged from sleep disorders, anxiety, and depression, to mania and psychotic disorders. They can be complicated by suicidal attempts and death by suicide.

We recommend that clinicians monitor psychiatric adverse effects in people treated with hydroxychloroquine, especially in the stressful context of the COVID19 pandemic. 


\section{Introduction}

Recent articles have raised concerns regarding the risk of psychological distress and psychiatric disorders, including suicidal behavior, surrounding the coronavirus disease 2019 (COVID-19) pandemic [1]. High levels of uncertainty, anxiety, social isolation, and economic adversity were mentioned as potential risk factors for psychiatric disorders and suicide attempts [2,3]. In the stressful context of the COVID-19 pandemic, concerns have emerged for the risk of psychiatric disorders with the use of hydroxychloroquine. On 14 May 2020, the Spanish drug agency warned of the risk of suicide with hydroxychloroquine for patients with COVID-19, after some reports of suicide emerged [4]; the French drug agency (Agence Nationale de sécurité du Médicament et des produits de santé; ANSM) also notified this risk [5].

The risk of psychiatric clinical manifestations during hydroxychloroquine treatment have already been reported but in the treatment of rheumatic diseases, systemic lupus erythematous, or malaria [6]. For example, some reports described patients with systemic lupus erythematous who developed suicidal ideation, self-harm, or acute psychosis after the introduction of hydroxychloroquine [7-10]. Thus, the widespread use of hydroxychloroquine for patients with COVID-19 may have also increased the risk of psychiatric symptoms.

To investigate this safety signal, we reviewed all psychiatric adverse effects with hydroxychloroquine in COVID19 patients registered in VigiBase, the World Health Organization's (WHO) global database of individual case safety reports, to 16 June 2020 . We investigated the risk of reporting psychiatric disorders with hydroxychloroquine compared with other drugs prescribed in COVID19 patients. For completeness, we sought to examine the psychiatric safety profile of hydroxychloroquine in other indications and before the COVID-19 pandemic.

\section{Methods}

\subsection{Data Sources}

The WHO global database VigiBase, maintained by the Uppsala Monitoring Centre (UMC), was used to gather anonymized safety reports. As at June 2020, VigiBase contained more than 22 million spontaneously generated adverse drug reactions, from 136 countries and covering more than $90 \%$ of the world's population [11, 12]. VigiBase includes information on patient's age, sex, medical history, country, and drugs taken, with their initiation and stop date. Reports are classified according the seriousness of the ADR. A serious adverse effect is defined as any effect leading to death, invalidation, significant incapacity, after effects, malformations, congenital anomalies, and birth defects, or that which requires hospitalization or prolongation of hospital stay. Each report contains variable amounts of information depending on the submitting countries. Each report originates from different sources, such as physicians, pharmacists, and other healthcare professionals, as well as non-healthcare professionals. Reports from clinical trials represent a small proportion of the reports found in VigiBase. All duplicate reports are identified using a statistical model and are removed from the database. The adverse effects are coded using the Medical Dictionary for Regulatory Activities (MedDRA ${ }^{\circledR}$ ). According to French law, Ethics Committee review was not necessary for this type of pharmacovigilance study. Additionally, because all data in VigiBase are anonymized, patient informed consent was also not required.

\subsection{Report Selection}

In a first analysis, we considered all reports with hydroxychloroquine classed as suspect or interacting and registered in VigiBase from 1 January 2020 to 16 June 2020. Psychiatric case reports were selected using the terminology 'Psychiatric disorders' in the System Organ Class (SOC) view found in MedDRA ${ }^{\circledR}$, or terms classified as 'Depression and suicide/self-injury' (broad) by the Standardized MedDRA ${ }^{\circledR}$ queries (SMQs) $[13,14]$. All psychiatric reports with hydroxychloroquine were then reviewed by one specialist in pharmacovigilance and clinical psychopharmacology (FM) to exclude those not related to a COVID-19 indication. We made the selection of COVID-19 reports based on the indication of hydroxychloroquine (or other drugs), including the terms 'COVID-19', 'COVID-19 respiratory', 'COVID-19 treatment', 'Coronavirus disease 2019', 'Coronavirus infection', 'Corona virus infection' or 'SARS-Cov-2 infection'. Finally, cases were classified into six groups of psychiatric disorders: insomnia or sleep disorders, anxiety, depression, psychotic disorders, suicide or self-injury, and cognitive disturbances. In a second analysis, we also reviewed all psychiatric reports of hydroxychloroquine registered from 1 January 1983 to 31 December 2019 for all patients $\geq 18$ years of age.

\subsection{Disproportionality Analysis}

In order to complete our pharmacovigilance review, we also performed a disproportionality analysis to compare cases of psychiatric disorders reported in COVID-19 patients exposed to hydroxychloroquine with those reported in COVID-19 patients exposed to remdesivir, tocilizumab, or 
lopinavir/ritonavir [15]. We used the case/non-case method, which is similar to case-control studies but adapted for pharmacovigilance studies [16]. We selected only reports with a drug prescribed for COVID-19. Within this selection, we identified cases of psychiatric adverse drug reactions (same selection as above). Non-cases were all other non-psychiatric adverse drug reactions registered with these drugs in VigiBase. To avoid reporting bias (notoriety bias) due to the Spanish safety alert, we considered only reports registered in VigiBase until 10 May 2020 [17].

We also performed another disproportionality analysis including reports registered in VigiBase after the Spanish safety alert (until 16 June 2020). We used reporting odds ratios (RORs) and their 95\% confidence intervals (CIs) to calculate disproportionality. The ROR is a ratio similar in concept to the odds ratio in case-control studies and corresponds to the exposure odds among reported cases of psychiatric disorders over the exposure odds among reported non-cases [18].

\section{Results}

\subsection{Psychiatric Safety of Hydroxychloroquine in Coronavirus Disease 2019 (COVID-19) Patients (2020)}

Among the 1754 reports with hydroxychloroquine in COVID-19 patients, we found 56 psychiatric adverse effects. Cases originated mainly from European countries (25) and from Morocco (19), involved mostly men ( 27 men, 25 women, 4 unknown), and had a mean age of 54.9 years (minimum 23 years, maximum 86 years) (Table 1). Hydroxychloroquine was prescribed at a dose of $400 \mathrm{mg} /$ day in $70 \%$ of reports (range $200-800 \mathrm{mg}$ ) and azithromycin was co-reported in 20 cases (36\%). Treatment duration with hydroxychloroquine varied between 1 day (discontinuation for serious adverse effects) and 27 days (median treatment duration 10 days). Twenty-eight
Table 1 Characteristics of psychiatric reports with hydroxychloroquine and other drugs used for coronavirus disease 2019, in Vigibase ${ }^{\mathrm{a}}$

\begin{tabular}{|c|c|c|}
\hline & $\begin{array}{l}\text { Hydroxychloroquine prescribed for } \\
\text { COVID- } 19^{\mathrm{a}}\end{array}$ & $\begin{array}{l}\text { Other drugs } \\
\text { prescribed for } \\
\text { COVID- } 19^{b}\end{array}$ \\
\hline Number of reports & 56 & 17 \\
\hline \multicolumn{3}{|l|}{ Reporting region } \\
\hline Europe & $25(45)$ & $11(65)$ \\
\hline Africa & $19(34)$ & 0 \\
\hline Asia & $9(16)$ & 0 \\
\hline Americas & $3(5)$ & $5(29)$ \\
\hline Oceania & 0 & $1(6)$ \\
\hline \multicolumn{3}{|l|}{ Reporting month } \\
\hline March & $1(2)$ & $2(12)$ \\
\hline April & $16(29)$ & $3(18)$ \\
\hline May & $32(57)$ & $5(29)$ \\
\hline Until 16 June & $7(13)$ & $7(41)$ \\
\hline \multicolumn{3}{|l|}{ Reporter } \\
\hline Physician & $27(48)$ & $8(47)$ \\
\hline Pharmacist or other healthcare professional & $24(43)$ & $6(35)$ \\
\hline Non-healthcare professional & $5(9)$ & $3(18)$ \\
\hline \multicolumn{3}{|l|}{ Sex } \\
\hline Women & $25(45)$ & $10(59)$ \\
\hline Men & $27(48)$ & $7(41)$ \\
\hline Unknown & $4(7)$ & 0 \\
\hline \multicolumn{3}{|l|}{ Age at onset, years } \\
\hline Mean (min-max) & $54.9(23-86)$ & $61.9(17-85)$ \\
\hline \multicolumn{3}{|l|}{ Serious } \\
\hline Yes & $28(50)$ & $10(59)$ \\
\hline
\end{tabular}

Data are expressed as $n(\%)$ unless otherwise specified

COVID-19 coronavirus disease 2019, min minimum, max maximum

${ }^{a}$ Until 16 June 2020

${ }^{\mathrm{b}}$ Remdesivir, tocilizumab, lopinavir/ritonavir 
cases were serious, with four completed suicides in men that occurred between 2 and 4 days after the first hydroxychloroquine prescription. In each suicide case, hydroxychloroquine was the sole suspected drug. In addition, we found three cases of intentional self-injury, 12 serious cases of psychotic disorders (mostly associated with visual hallucinations, agitations, or aggressions), 7 cases of insomnia or anxiety, and 2 cases of confusions. Among non-serious cases, insomnia or anxiety symptoms were mostly described (19). No cases of intentional self-injury or suicide were reported with remdesivir, tocilizumab, or lopinavir/ritonavir during the study period. Most psychiatric cases reported with remdesivir, tocilizumab, or lopinavir/ritonavir related to anxiety or agitation (10 cases, $59 \%)$.

Compared with remdesivir, tocilizumab, or lopinavir/ritonavir, the use of hydroxychloroquine was associated with an increased risk of reporting psychiatric disorders in treating COVID-19 (ROR 6.27, 95\% CI 2.74-14.35) (Table 2).

\subsection{Review of Psychiatric Reports Recorded in VigiBase (Until 2019)}

We found 751 psychiatric adverse drug reactions reported in VigiBase before 2020. Psychiatric reports were mainly insomnia or sleep disorders (32\%), followed by anxiety symptoms (22\%), depression (20\%), and psychotic disorders (14\%) (Table 3). Most of the cases related to women (82\%), and the mean age reported was 53.5 years. The main psychotropic drugs co-reported were antidepressants (19.7\%). The majority of adverse drug reactions were reported as serious (59\%). Completed suicide (48) was the main cause of death among all adverse drug reactions reported with hydroxychloroquine, followed by cardiac adverse effects (cardiomyopathy) and respiratory failure. The median time to onset of psychiatric effects with hydroxychloroquine was 5 days.

\section{Discussion}

While studies suggested not only an absence of therapeutic benefit for COVID-19 patients but also potential cardiac harms with the use of hydroxychloroquine, our analysis also raises concerns about the psychiatric safety of hydroxychloroquine for COVID-19 patients [19, 20]. This pharmacovigilance analysis suggests that some COVID-19 patients exposed to hydroxychloroquine experienced serious psychiatric disorders, and, among these patients, some committed suicide. We also found in the review of reports made before 2020 that among all adverse drug reactions reported with hydroxychloroquine, completed suicide was the first cause of death.

Psychiatric clinical manifestations were reported with hydroxychloroquine in other indications such as rheumatic diseases, systemic lupus erythematous, or malaria. The first description of hydroxychloroquine inducing psychiatric effects was made in 1962 with a case-series of psychological symptoms and depression in patients treated for rheumatoid diseases [21]. Thereafter, several reports of mood disorders (including suicidal behavior), suicide, and psychotic disorders were published [7-10]. While several pharmacodynamic mechanisms were hypothesized to explain these psychiatric adverse effects (including serotonin imbalance), no clear explanation could be retained [22]. In the context of autoimmune disease or malaria, some factors have been associated with psychiatric adverse drug reactions, such as history of psychiatric disorders, female sex, low body weight, or alcohol abuse. High doses of hydroxychloroquine
Table 2 Reporting odds ratios for the association between psychiatric disorder reports and the use of hydroxychloroquine for coronavirus disease 2019, in Vigibase

\begin{tabular}{lccc}
\hline Exposures & Cases $^{\mathrm{a}}$ & Non-cases $^{\mathrm{b}}$ & ROR (95\% CI) \\
\hline $\begin{array}{l}\text { Analysis including only cases reported before the Spanish } \\
\text { safety alert }\end{array}$ & & & \\
Other drugs prescribed for COVID-19 & & & \\
Hydroxychloroquine & 7 & 513 & 1 (reference) \\
Analysis including all cases reported until 16 June 2020 & 31 & 726 & $6.27(2.74-14.35)$ \\
Other drugs prescribed for COVID-19 & 17 & 1280 & 1 (reference) \\
Hydroxychloroquine & 56 & 1698 & $2.48(1.43-4.29)$ \\
\hline
\end{tabular}

CI confidence interval, COVID-19 coronavirus disease 2019, ROR reporting odds ratio, SOC System Organ Class, MedDRA Medical Dictionary for Regulatory Activities

${ }^{a}$ Cases were individual case safety reports containing any terms including the terminology 'Psychiatric disorders' in the SOC view found in MedDRA ${ }^{\circledR}$

${ }^{b}$ Non-cases were individual case safety reports containing all other adverse events reported to be linked with the respective drug

${ }^{c}$ Cases reported until 10 May 2020

${ }^{\mathrm{d}}$ Remdesivir, tocilizumab, lopinavir/ritonavir 
Table 3 Characteristics of psychiatric reports with hydroxychloroquine registered in VigiBase until December 2019

\begin{tabular}{|c|c|c|c|c|c|c|c|}
\hline & $\begin{array}{l}\text { Psychiatric } \\
\text { adverse } \\
\text { effects }^{\mathrm{a}}\end{array}$ & $\begin{array}{l}\text { Insomnia or } \\
\text { sleep disor- } \\
\text { ders }\end{array}$ & Anxiety & Depression & Psychotic disorders & $\begin{array}{l}\text { Suicide or self- } \\
\text { injury }\end{array}$ & $\begin{array}{l}\text { Cognitive } \\
\text { disturbances }\end{array}$ \\
\hline Number of reports & 751 & 237 & 166 & 148 & 104 & 92 & 74 \\
\hline \multicolumn{8}{|l|}{ Reporting region } \\
\hline Americas & $484(64.5)$ & $152(64.1)$ & $116(69.9)$ & $100(67.6)$ & $52(50.0)$ & $67(72.8)$ & $41(55.4)$ \\
\hline Europe & $194(25.8)$ & $47(19.8)$ & $39(23.5)$ & $45(30.4)$ & $43(41.4)$ & $22(23.9)$ & $29(39.2)$ \\
\hline Others $^{\mathrm{b}}$ & $73(9.7)$ & $38(16.0)$ & $11(6.6)$ & d & $9(8.6)$ & d & d \\
\hline \multicolumn{8}{|l|}{ Reporting year } \\
\hline 1983-1999 & $42(5.6)$ & d & $9(5.4)$ & $8(5.4)$ & $13(12.5)$ & d & $9(12.2)$ \\
\hline 2000-2009 & $73(9.7)$ & $18(7.6)$ & $18(10.9)$ & $17(11.5)$ & $11(10.6)$ & d & $8(10.8)$ \\
\hline 2010-2019 & $635(84.7)$ & $215(90.7)$ & $139(83.7)$ & $123(83.1)$ & $80(76.9)$ & $86(93.5)$ & $57(77.0)$ \\
\hline \multicolumn{8}{|l|}{ Reporter } \\
\hline Physician & $312(41.5)$ & $96(40.5)$ & $65(39.2)$ & $67(45.3)$ & $47(45.2)$ & $44(47.8)$ & $32(43.2)$ \\
\hline Others $^{\mathrm{c}}$ & $340(45.3)$ & $111(46.8)$ & $80(48.2)$ & $65(43.9)$ & $37(35.6)$ & $40(43.5)$ & $29(39.2)$ \\
\hline Unknown & $99(13.2)$ & $30(12.7)$ & $21(12.7)$ & $16(10.8)$ & $20(19.2)$ & $8(8.7)$ & $13(17.6)$ \\
\hline \multicolumn{8}{|l|}{ Sex } \\
\hline Women & $617(82.2)$ & $196(82.7)$ & $145(87.4)$ & $123(83.1)$ & $86(82.7)$ & $72(78.3)$ & $63(85.1)$ \\
\hline \multicolumn{8}{|l|}{ Age at onset, years } \\
\hline Mean \pm SD & $53.5(16.0)$ & $55.0(14.9)$ & $52.8(15.0)$ & $55.2(14.6)$ & $53.8(17.4)$ & $49.4(19.7)$ & $55.3(17.5)$ \\
\hline \multicolumn{8}{|l|}{ Time to onset, days } \\
\hline Median (IQR) & $5(0-44)$ & $1(0-8)$ & $5(0-227)$ & $7(0-36)$ & $7.5(4-110)$ & d & $7(5-83)$ \\
\hline \multicolumn{8}{|l|}{ Serious } \\
\hline Yes & $443(59.0)$ & $125(52.7)$ & $90(54.2)$ & $94(63.5)$ & $68(65.4)$ & $85(92.4)$ & $47(63.5)$ \\
\hline \multicolumn{8}{|l|}{ Evolution } \\
\hline Death & $64(8.5)$ & 0 & 1 & 1 & 0 & $48(52.2)$ & 0 \\
\hline \multicolumn{8}{|l|}{ Indications } \\
\hline $\begin{array}{l}\text { Rheumatoid } \\
\text { arthritis }\end{array}$ & $222(29.6)$ & 89 (37.6) & $50(30.1)$ & $53(35.8)$ & $19(18.3)$ & $5(5.4)$ & $12(16.2)$ \\
\hline Other arthritis & $43(5.7)$ & $15(6.3)$ & $12(7.2)$ & $7(4.7)$ & $6(5.8)$ & d & $7(9.5)$ \\
\hline $\begin{array}{l}\text { Systemic lupus } \\
\text { erythematosus }\end{array}$ & $41(5.5)$ & $10(4.2)$ & $7(4.2)$ & d & $8(7.7)$ & $6(6.5)$ & $5(6.8)$ \\
\hline $\begin{array}{l}\text { Other auto-immune } \\
\text { diseases }\end{array}$ & $44(5.9)$ & $14(5.9)$ & $13(7.8)$ & $8(5.4)$ & $7(6.7)$ & $6(6.5)$ & $5(6.8)$ \\
\hline Other indications & $35(4.7)$ & $6(2.5)$ & $6(3.6)$ & d & $11(10.6)$ & $8(8.7)$ & $5(6.8)$ \\
\hline Unknown & $366(48.6)$ & $103(43.5)$ & $78(47.0)$ & $71(48.0)$ & $53(50.9)$ & $63(68.5)$ & $40(53.9)$ \\
\hline \multicolumn{8}{|l|}{$\begin{array}{l}\text { Psychotropic drugs } \\
\text { co-reported }\end{array}$} \\
\hline Antidepressants & 148 (19.7) & $31(13.1)$ & $34(20.5)$ & $48(32.4)$ & $25(24.0)$ & $25(27.2)$ & $23(31.1)$ \\
\hline $\begin{array}{l}\text { Anxiolytics/hyp- } \\
\text { notics }\end{array}$ & $107(14.3)$ & $25(10.6)$ & $21(12.7)$ & 32 (21.6) & $26(25.0)$ & $26(28.3)$ & $23(31.1)$ \\
\hline Antipsychotics & $43(5.7)$ & $6(2.5)$ & $8(4.8)$ & $23(15.5)$ & $21(20.2)$ & $6(6.5)$ & $18(24.3)$ \\
\hline Antiepileptics & 85 (11.3) & $29(12.2)$ & $24(8.4)$ & $29(19.6)$ & $26(25.0)$ & $11(11.9)$ & $21(28.4)$ \\
\hline
\end{tabular}

Data are expressed as $n(\%)$ unless otherwise specified

SOC System Organ Class, MedDRA Medical Dictionary for Regulatory Activities, SMQs Standardized MedDRA® queries, $S D$ standard deviation, $I Q R$ interquartile range

${ }^{a}$ Psychiatric adverse effects were selected using the terminology 'Psychiatric disorders' in the SOC view found in MedDRA®, or terms classified as 'Depression and suicide/self-injury' (broad) by the SMQs. Psychiatric adverse effects included the subgroups 'Insomnia or sleep disorders', 'Anxiety', 'Depression', 'Psychotic disorders', and 'Cognitive disturbances', including 'Suicide or self-injury'. Each subgroup was not mutually exclusive

${ }^{\mathrm{b}}$ Oceania and Asia

${ }^{c}$ Other healthcare professionals and non-healthcare professionals (patient, pharmaceutical industry)

${ }^{\mathrm{d}}$ Insufficient reports to calculate (fewer than five) 
and use of concomitant cytochrome P450 (CYP) 3A4 inhibitors have been also identified as potential risk factors [23]. Our analysis of cases reported before 2020 is in line with these previous publications, as psychiatric reports were mainly insomnia or anxiety, related to mainly women, and the median time to onset was 5 days. In the context of COVID-19, we found similar characteristic of psychiatric reports, except for sex as most cases involved men. The sex difference can be explained by a higher proportion of men treated by hydroxychloroquine due to a higher prevalence of COVID-19 complications in men [24]. Finally, it should also be underlined that the proportions of reports of suicide or self-injury were similar in COVID-19 indications than other indications (12\%).

While our results are suggestive, for the first time, of an increased risk of reporting psychiatric disorders with hydroxychloroquine in COVID-19 indications, this risk must be put into context with the strengths and limitations of pharmacovigilance studies in order to understand its significance. Since our study was conducted in VigiBase, we could not control our analyses on disease severity, as this clinical data is not included in reports in the WHO database. Therefore, no causal relation can be made on the risk of psychiatric disorders with hydroxychloroquine versus remdesivir, tocilizumab, or lopinavir/ritonavir. In fact, we could not exclude that patients exposed to remdesivir, tocilizumab, or lopinavir/ritonavir were less likely to develop psychiatric symptoms than patients exposed to hydroxychloroquine. While the disproportionality analysis is susceptible to indication bias, the relatively high number of psychiatric reports with hydroxychloroquine should be discussed. From a methodological point of view, the outcome validity of adverse drug reactions registered in pharmacovigilance databases such as VigiBase should be underlined. Case reports in pharmacovigilance databases should not just be considered mere lines of pharmacoepidemiologic data, because, individually, each line really represents a true clinical event with a precise medical meaning [25]. Moreover, reports recorded in pharmacovigilance databases have been found to be particularly useful for generating or testing hypotheses [26]. Therefore, reporters may have taken into account potential confounding factors as the stressful context of the COVID19 pandemic when they reported psychiatric disorders with hydroxychloroquine.

Of note, the safety signal emerged while there was a potential notoriety bias for another specific adverse drug reaction with hydroxychloroquine. Indeed, during the study period, research articles have suggested a potential risk of cardiac disorders with hydroxychloroquine alone or associated with azithromycin in COVID-19 patients. On 24 April 2020, the US FDA warned that hydroxychloroquine can cause serious heart rhythm disorders in patients with COVID-19, often in combination with azithromycin and other drugs causing QT prolongation [27]. Notoriety bias is defined as "a selection bias in which a case has a greater chance of being reported (cardiac disorders) if the subject is exposed to the studied factor known to cause (hydroxychloroquine), thought to cause, or likely to cause the event of interest" [28].

However, our study does come with several important strengths. We used the largest pharmacovigilance database in the world, which covers more than $90 \%$ of the world's population, allowing us to detect and follow potential safety signals during the COVID-19 pandemic crisis. These results from a globalized database increase the external validity of our findings. Second, working with this data from VigiBase allows us to be in the context of 'real-world practice', unlike clinical trials. Third, the case/ non-case method performed in such pharmacovigilance databases allowed the evaluation of rare adverse drug reactions, such as psychiatric disorders with hydroxychloroquine. Unlike cohort studies, only a few reports of exposed cases are needed to carry out this type of analysis. Fourth, in regard to the outcomes, we expect minimal misclassification and good validity in the diagnosis of psychiatric disorders. Finally, our findings corroborate the previous signal of psychiatric disorders with hydroxychloroquine in other indications (rheumatic diseases or malaria).

\section{Conclusion}

Our findings imply that during the COVID-19 pandemic, some patients may have experienced psychiatric symptoms associated with the use of hydroxychloroquine. The risk of psychiatric disorders with hydroxychloroquine is probably a rare adverse drug reaction, but could be serious as several cases of completed suicide were reported. Given the paucity of evidence of benefit in COVID-19, as well as this signal of psychiatric risk, there is no reason to prescribe hydroxychloroquine for treating COVID-19 patients in routine practice [19]. In addition, psychiatric symptoms should be monitored when prescribing hydroxychloroquine in clinical trials. Further real-world studies are needed to quantify the psychiatric risk associated with hydroxychloroquine during the COVID-19 pandemic.

Acknowledgements The authors acknowledge the assistance of the UMC, which provided and gave permission to use the data analyzed in the present study. VigiBase ${ }^{\circledR}$ is freely available in our department, which is part of the WHO pharmacovigilance network. The authors are indebted to the National Pharmacovigilance Centres that contributed data. The opinions and conclusions in this study are not necessarily those of the various centres or of the WHO or ANSM (France). 


\section{Declarations}

Conflicts of Interest Philippe Garcia, Alexis Revet, Antoine Yrondi, Vanessa Rousseau, Yannick Degboe, and François Montastruc have no conflicts of interest to declare.

Funding This work was performed during the university research period of the authors, using the database that is available without fees in the department of the authors.

Ethics Approval According to the French law, review from an ethics committee was not necessary for this type of pharmacovigilance study.

Consent to Participate Because all data in VigiBase are anonymized, patient informed consent is also not required.

Consent for Publication Not applicable.

Availability of Data and Material Anonymized data can be made available to investigators upon request to the corresponding author.

Code Availability Not applicable.

Author Contributions Dr. François Montastruc had full access to all of the data in this study and takes responsibility for the integrity of the data and the accuracy of the data analysis. Concept and design: Philippe Garcia and François Montastruc. Acquisition, analysis, or interpretation of the data: All authors. Drafting of the manuscript: François Montastruc. Critical revision of the manuscript for important intellectual content: All authors. Statistical analyses: Vanessa Rousseau. Supervision: François Montastruc.

\section{References}

1. Gunnell D, Appleby L, Arensman E, Hawton K, John A, Kapur $\mathrm{N}$, et al. Suicide risk and prevention during the COVID-19 pandemic. Lancet Psychiatry. 2020;7:468-71.

2. Kawohl W, Nordt C. COVID-19, unemployment, and suicide. Lancet Psychiatry. 2020;7:389-90.

3. Reger MA, Stanley IH, Joiner TE. Suicide mortality and coronavirus disease 2019: a perfect storm? JAMA Psychiatry. 2020. https://doi.org/10.1001/jamapsychiatry.2020.1060.

4. Cloroquina/Hidroxicloroquina: precauciones y vigilancia de posibles reacciones adversas en pacientes con COVID-19. Agencia Española de Medicamentos y Productos Sanitarios. 2020. https://www.aemps.gob.es/informa/notasinformativas/ medicamentosusohumano-3/seguridad-1/2020-seguridad-1/ cloroquina-hidroxicloroquina-precauciones-y-vigilancia-deposibles-reacciones-adversas-en-pacientes-con-covid-19/. Accessed 25 June 2020

5. Médicaments utilisés chez les patients atteints du COVID19: une surveillance renforcée des effets indésirables-Point d'information (actualisé le 14/05/2020)_ANSM: Agence nationale de sécurité du médicament et des produits de santé. https://ansm.sante.fr/S-informer/Actualite/Medicaments-utili ses-chez-les-patients-atteints-du-COVID-19-une-surveillan ce-renforcee-des-effets-indesirables-Point-d-information-actua lise-le-14-05-2020. Accessed 25 Jun 2020

6. de Ribeiro NPO, de Schier ARM, Ornelas AC, de Oliveira CM, Nardi AE, Silva AC. Anxiety, depression and suicidal ideation in patients with rheumatoid arthritis in use of methotrexate, hydroxychloroquine, leflunomide and biological drugs. Compr Psychiatry. 2013;54:1185-9.

7. Gonzalez-Nieto JA, Costa-Juan E. Psychiatric symptoms induced by hydroxychloroquine. Lupus. 2015;24:339-40.

8. Good MI, Shader RI. Behavioral toxicity and equivocal suicide associated with chloroquine and its derivatives. Am J Psychiatry. 1977;134:798-601.

9. Manzo C, Gareri P, Castagna A. Psychomotor agitation following treatment with hydroxychloroquine. Drug Saf Case Rep. 2017;4:6.

10. Das P, Rai A, Chopra A, Philbrick K. Psychosis likely induced by hydroxychloroquine in a patient with chronic $\mathrm{Q}$ fever: a case report and clinically relevant review of pharmacology. Psychosomatics. 2014;55:409-13.

11. Lindquist M. VigiBase, the WHO global ICSR database system: basic facts. Drug Inf J. 2008;42:409-19.

12. UMC I VigiBase. https://www.who-umc.org/vigibase/vigibase/. Accessed 4 Mar 2020

13. MedDRA MSSO Welcome. https://www.meddramsso.com/. Accessed 4 June 2013

14. Brown EG, Wood L. The medical dictionary for regulatory activities (MedDRA). Drug Saf. 1999;20(2):109-17.

15. Sanders JM, Monogue ML, Jodlowski TZ, Cutrell JB. Pharmacologic treatments for coronavirus disease 2019 (COVID-19): a review. JAMA. 2020;323(18):1824-36.

16. Faillie J-L. Case-non-case studies: principle, methods, bias and interpretation. Therapie. 2019;74:225-32.

17. Pariente A, Gregoire F, Fourrier-Reglat A, Haramburu F, Moore $\mathrm{N}$. Impact of safety alerts on measures of disproportionality in spontaneous reporting databases: the notoriety bias. Drug Saf. 2007;30:891-8.

18. Rothman KJ, Lanes S, Sacks ST. The reporting odds ratio and its advantages over the proportional reporting ratio. Pharmacoepidemiol Drug Saf. 2004;13:519-23.

19. Fiolet T, Guihur A, Rebeaud M, Mulot M, Peiffer-Smadja N, Mahamat-Saleh Y. Effect of hydroxychloroquine with or without azithromycin on the mortality of COVID-19 patients: a systematic review and meta-analysis. Clin Microbiol Infect. 2020. https://doi.org/10.1016/j.cmi.2020.08.02 (Epub 26 Aug 2020).

20. Gérard A, Romani S, Fresse A, Viard D, Parassol N, Granvuillemin A, et al. "Off-label" use of hydroxychloroquine, azithromycin, lopinavir-ritonavir and chloroquine in COVID-19: a survey of cardiac adverse drug reactions by the French Network of Pharmacovigilance Centers. Therapie. 2020;75:371-9.

21. Drew J. Concerning the side effects of antimalarial drugs used in the extended treatment of rheumatic disease. Med J Aus. 1962;618-20.

22. Mascolo A, Berrino PM, Gareri P, Castagna A, Capuano A, Manzo C, et al. Neuropsychiatric clinical manifestations in elderly patients treated with hydroxychloroquine: a review article. Inflammopharmacology. 2018;26:1141-9.

23. Ferraro V, Mantoux F, Denis K, Lay-Macagno M-A, Ortonne J-P, Lacour J-P. Hallucinations during treatment with hydrochloroquine. Ann Dermatol Venereol. 2004;131:471-3 (in French).

24. Huang C, Wang Y, Li X, Ren L, Zhao J, Hu Y, et al. Clinical features of patients infected with 2019 novel coronavirus in Wuhan, China. Lancet. 2020;395:497-506.

25. Aronson JK. Case reports as evidence in pharmacovigilance. Mann's pharmacovigilance. New York: Wiley; 2014. p. 121-37.

26. Aronson JK. Post-marketing drug withdrawals: pharmacovigilance success, regulatory problems. Therapie. 2017;72:555-61.

27. Center for Drug Evaluation and Research. FDA cautions against use of hydroxychloroquine or chloroquine for COVID-19 outside of the hospital setting or a clinical trial due to risk of heart 
rhythm problems. FDA. 2020. https://www.fda.gov/drugs/drugsafety-and-availability/fda-cautions-against-use-hydroxychl oroquine-or-chloroquine-covid-19-outside-hospital-setting-or. Accessed 25 June 2020

28. de Boissieu P, Kanagaratnam L, Abou Taam M, Roux M-P, Dramé $\mathrm{M}$, Trenque T. Notoriety bias in a database of spontaneous reports: the example of osteonecrosis of the jaw under bisphosphonate therapy in the French national pharmacovigilance database. Pharmacoepidemiol Drug Saf. 2014;23:989-92. 